\title{
Electron field emission from amorphous silicon
}

\author{
S. R. P. Silva, ${ }^{\text {a) }}$ R. D. Forrest, J. M. Shannon, and B. J. Sealy \\ School of Electronic Engineering, Information Technology and Mathematics, University of Surrey, \\ Guildford, Surrey GU2 5XH, United Kingdom
}

(Received 19 July 1998; accepted 7 December 1998)

\begin{abstract}
Hydrogenated amorphous silicon $(a-\mathrm{Si}: \mathrm{H})$ is used commercially for large area device fabrication in active matrix flat panel displays as the switching element in thin film transistors. We have found that $a-\mathrm{Si}: \mathrm{H}$ thin films can be conditioned to field emit electrons at relatively low electric fields. Once conditioned, emission from nonoptimized films at macroscopic vacuum fields below $20 \mathrm{~V} / \mu \mathrm{m}$ is routinely obtained. The threshold fields for electron emission appears to be dependent on the film thickness and fields below $10 \mathrm{~V} / \mu \mathrm{m}$ have also been recorded. The Schottky junction between the $a$-Si:H and $\mathrm{Cr}$ is important to the electron emission process at low fields and plays a major role in helping to fully deplete the $a$-Si:H film such that electrons that enter the conduction band of the film by thermionic emission over the barrier (or tunneling through the barrier) can become "hot." If the emission is localized, it still appears to be controlled by space charge and the back contact barrier. We have examined the electron emission from multilayer structures that contain Schottky and ohmic back contacts as well as thin $n^{+}$surface layers. Although the electron emission can be fitted to a Fowler-Nordheim type curve, the values obtained for the barrier and emission area for the nominally flat thin films are unrealistic. We explain the observed emission using a space charge induced hot electron model, where the $a$-Si:H acts as an interlayer in which the electrons are heated.

(c) 1999 American Vacuum Society. [S0734-211X(99)00702-7]
\end{abstract}

\section{INTRODUCTION}

The properties of hydrogenated amorphous silicon ( $a$ $\mathrm{Si}: \mathrm{H})$ thin films have been studied for a number of years, and currently it is the only widely used large area amorphous semiconductor for electronic applications. ${ }^{1}$ Despite its low electron mobility, it is used both for passive and active devices, especially in the flat panel displays area. Last year we reported the experimental observation of electron field emission from flat $a$-Si:H films at relatively low electric fields. ${ }^{2}$ This was surprising due to the fact that the measured electron affinity of these films is of the order of $4 \mathrm{eV}$, and, therefore it would be difficult to envisage that electron emission can take place from these nominally flat films at low fields purely due to a Fowler-Nordheim like tunneling mechanism. The Fowler-Nordheim model states that electrons can leave the surface of a metal into vacuum due to the tunneling of electrons from the Fermi level of the metal into the vacuum by the application of a high electric field. These fields must be of the order of thousands of volts per micron for emission to take place from flat films that have a typical electron affinity of $4 \mathrm{eV}$. Therefore, in order to obtain emission from metal surfaces, tips with high curvatures or high beta factors need to be fabricated using microlithographic techniques. ${ }^{3}$ Thus, the holy grail for cathode manufacturers has been the production of a large area stable flat cathode that emits electrons at relatively low electric fields. If the emission is governed by the Fowler-Nordheim tunneling, this could only occur if the films have a low electron affinity or sharp features (high beta) that would increase the localized electric field experienced by the electrons due to geometric considerations.

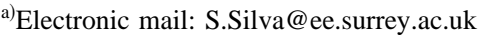

Amorphous carbon $(a-C)$ and polycrystalline diamond thin films have been shown to emit electrons from nominally flat films at low electric fields. ${ }^{4-7}$ Based on these results major industrial effort has been expended on researching these flat cathodes and it has been announced that they will probably be used for the next generation of electron emitters in field emission flat panel displays that are currently based on Spindt tip emitters. ${ }^{8}$ The emission obtained from the $a$-Si:H thin films reported in this article are comparable to those obtained from the diamond-like carbon (DLC) thin films. Since $a$-Si technology is at a significantly more advanced state than DLC, it is envisaged that these films hold considerable promise as a practical cathode material for flat panel displays. The use of $a-\mathrm{Si}: \mathrm{H}$ as a cathode is further supported by the ability of $a-\mathrm{Si}: \mathrm{H}$ to form active components such as thin film transistors, which will help in the fabrication of three terminal devices, which would be necessitated if the current in the cold cathode were to be space charge limited. $^{4}$

\section{EXPERIMENT}

The $a$-Si:H thin films were grown using $\mathrm{SiH}_{4}$ and $\mathrm{H}_{2}$ as feed gases in a standard plasma enhanced chemical vapor deposition system at a temperature of $250{ }^{\circ} \mathrm{C}$ and a growth rate of $\sim 25 \AA / \mathrm{min}$. The films were undoped, and deposited on glass substrates coated with $\mathrm{Cr}(50 \mathrm{~nm})$. The $\mathrm{Cr}$ films were continuous with no evidence of island growth. The $a$-Si:H films were of the quality used to produce switching elements for thin film transistors/diodes in matrix-addressed flat panel displays. The hydrogen content in the deposited $a$-Si:H films was around 10 at. \%. The films have an optical Tauc gap of $1.74 \mathrm{eV}$. The "gap" state defect density is ex- 


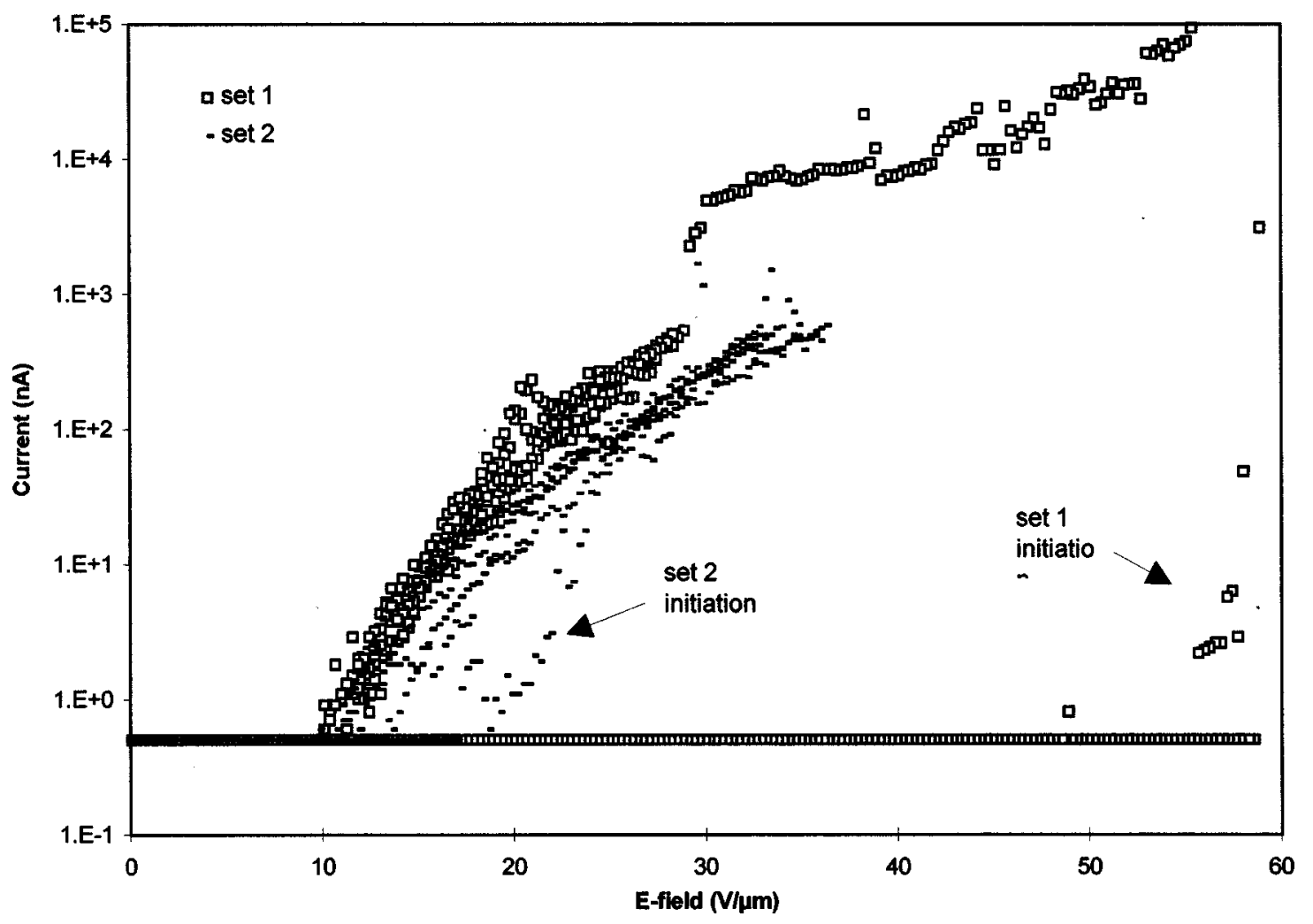

FIG. 1. Typical electron field emission characteristic showing the need for an initiation field to be applied before reproducible $I-E$ characteristics are obtained. The data in set 2 was obtained after exposing the 100-nm-thick $a$-Si:H film to air and remeasuring the $I-E$ characteristics.

pected to be below $10^{16} \mathrm{~cm}^{-3} \mathrm{eV}^{-1}$ in the middle of the gap. The films are slightly $n$ type due to a structural (bonding) defect present in the $a$-SiH films.

For field emission measurements a spherical stainless steel anode, $5 \mathrm{~mm}$ in diameter was placed above the cathode surface which was greater than $20 \mathrm{~mm} \times 20 \mathrm{~mm}$ in area, by means of a micrometer-controlled apparatus. The current $(I)$ versus voltage $(V)$ was obtained at a vacuum better than 5 $\times 10^{-6}$ mbar. The electric field discussed in this article are macroscopic fields applied to a vacuum gap of $40 \mu \mathrm{m}$. Electron emission was not observed from flat single crystal $n^{+}-\mathrm{Si}$ $(0.1 \Omega \mathrm{cm})$ substrates at fields as high as $120 \mathrm{~V} / \mu \mathrm{m}$, the limit of our apparatus. Reverse leakage currents were measured after each experiment. Reverse leakage currents were less than the minimum detectable limit of $1 \times 10^{-9} \mathrm{~A}$ for the measurement system used. Each current recorded is the average of ten measurements at the fixed bias, with a fixed delay period between each measurement of $2 \mathrm{~s}$. The voltage was then ramped slowly to the next value, and the next measurement taken after a delay of $60 \mathrm{~s}$. The electric field $(E)$ shown in Figs. 1, 2, and 3, is calculated simply by dividing the applied voltage $(V)$ by the anode cathode spacing $(d)$. As shown in Fig. 1, an initiation field has to be applied to the cathodes to turn them on. Thereafter, the threshold field at which the emission takes place from the cathodes can be reduced by cycling the electric field over two to three periods. This we refer to as conditioning. If during this conditioning period we increase the current emitted above a cur- rent threshold limit for a prolonged period of time, there results a noticeable change in surface morphology. These films that show surface morphological changes we refer to as stressed films, see Fig. 4. The reverse leakage current for all the results shown in this study remained below the detection limit of our measurement system.

\section{RESULTS AND DISCUSSION}

The variation of a typical $(I-E)$ characteristic showing the initiation process and conditioning is shown in Fig. 1. The data marked as set 1 in Fig. 1 shows three cycles of emission current readings obtained when the voltage was cycled three times between preset current limits. The variation shown is for a $100-\mathrm{nm}$-thick $a-\mathrm{Si}: \mathrm{H}$ film, which after initiation follows a fixed $I-E$ characteristic quite reproducibly. The data referred to as set 2 in Fig. 1 was obtained from the same cathode region that had been let up to air for several hours and the retested under the same conditions after a period of $24 \mathrm{~h}$. It is noticeable that the initiation that is required in the second run is significantly lower than the first run, and is tending towards the steady state values despite being exposed to air. The $I-E$ curve obtained for set 2 matches that obtained for set 1 quite closely.

Once the cathode has been initiated, and conditioned, the exponentially rising region of the field emission current is followed by what almost looks like a superlinear region. This would be expected if the current were to be bulk film limited 


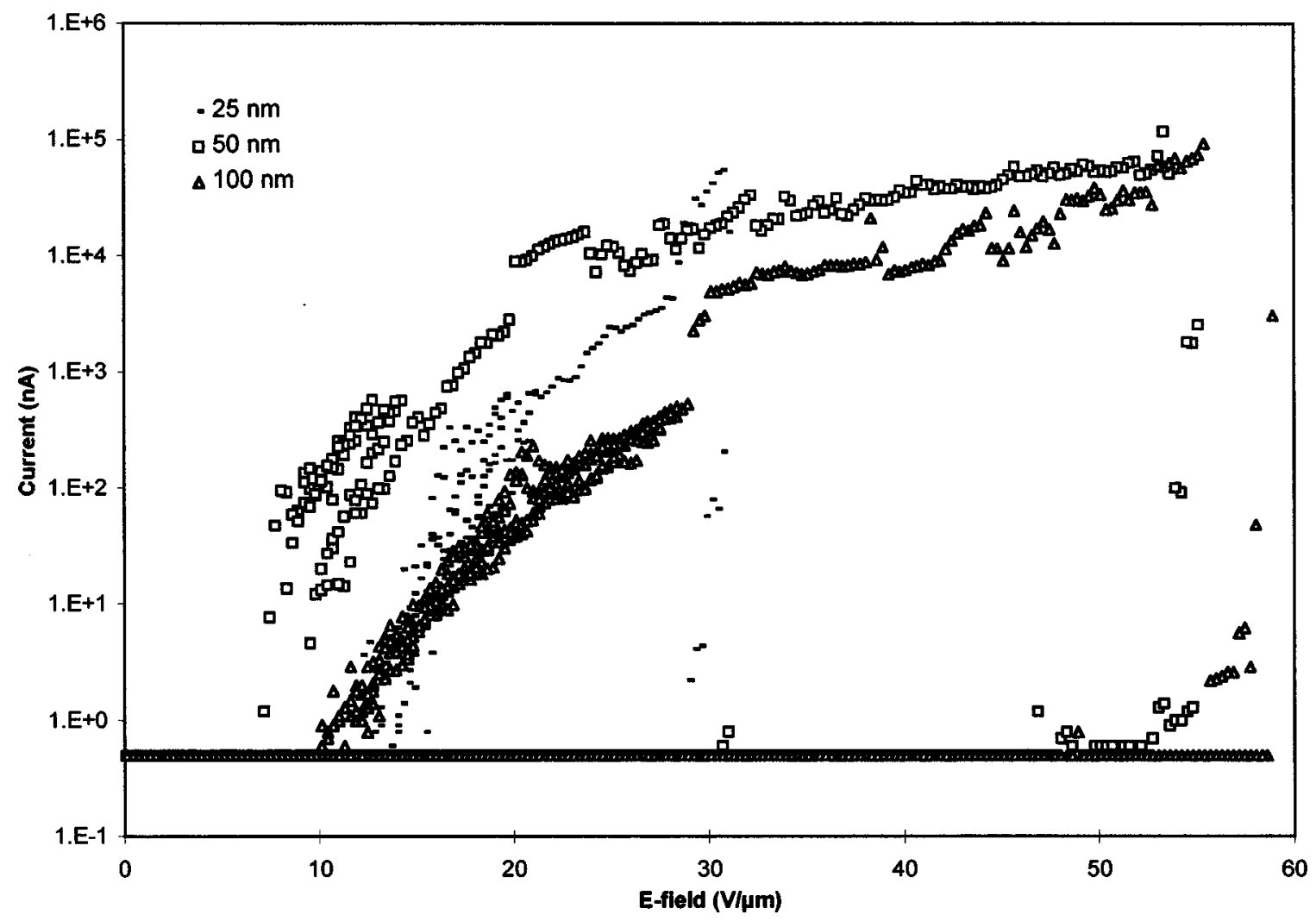

FIG. 2. Variation of the $I-E$ curves as a function of film thickness. The $a-\mathrm{Si}: \mathrm{H}$ films with thicknesses of 25,50 , and $100 \mathrm{~nm}$ are used to obtain the emission.

as expected from a space-charge-limited current process through the thin film. The exponential turn-on region remains for over three orders of magnitude, spread over 20 $\mathrm{V} / \mu \mathrm{m}$, and then shows a much reduced increase in field emission current with electric field. The threshold field for this cathode is of the order of $10 \mathrm{~V} / \mu \mathrm{m}$, which is comparable to those observed for undoped diamond-like carbon thin films. ${ }^{9,10}$ The variation observed as a function of film thickness for the $a-\mathrm{Si}: \mathrm{H}$ films, where $I-E \mathrm{~s}$ both for before and after initiation appear in Fig. 2. The threshold field appears to first decrease and then increase with decreasing film thickness, a feature that should remain approximately constant if the emission was purely a surface effect as expected for Fowler-Nordheim-like tunneling into vacuum. All the films show an exponentially increasing current region of greater than three orders of magnitude followed by a much shallower increase. In addition, the initial rate of increase is different for the three films analyzed with the thickest film showing the least emission current increase per unit increase in electric field and the thinnest film showing the highest slope. Both the variation of threshold field and rate of increase would be expected for films whose electron emission properties were primarily controlled by band bending in the films and therefore would be critically dependent upon the thickness' of films that controlled its space charge capability. The field required to obtain emission from the cathodes may be of significance as well. The initiation field increases as a function of film thickness indicating the need for higher fields to penetrate fully into the films before they become fully depleted. Latham ${ }^{11}$ first proposed the use of a "hot electron emission model" to explain emission from insulating materials, which was later modified to incorporate electron emission from semiconductors as a result of space charge induced band bending which gives a thickness dependent electric field, ${ }^{4}$ as seen in these films. It is possible that the emission observed in these films are localized as no site density information is available as yet. But, it is unlikely to be a result of breakdown as inferred by scanning electron microscopy (SEM) analysis. If the emission is localized, it too appears to be controlled by the space charge and the back contact barrier.

All the films discussed in Fig. 2 have Schottky barriers present between the $\mathrm{Cr}$ metal back contact and the $a-\mathrm{Si}: \mathrm{H}$ thin film. As a result, the barrier present at the back contact would block the flow of charge into the thin $a$-Si:H films until the band bending in the films due to space-charge considerations is very large. The electrons that then thermionically overcome the barrier (and/or tunnel through) at the back contact and enter the conduction band of the $a-\mathrm{Si}: \mathrm{H}$ film will gather much kinetic energy while traversing down the electric field towards the front surface of the cathode, in the process becoming hot. It is then possible that close to the front surface of the cathodes the energy of these hot electrons will be great enough to overcome the surface electron affinity and thereby leave the film as field emitted electrons.

The emission current observed experimentally in the films, although following a Fowler-Nordheim type tunneling current equation, did not give realistic values for the fitting 


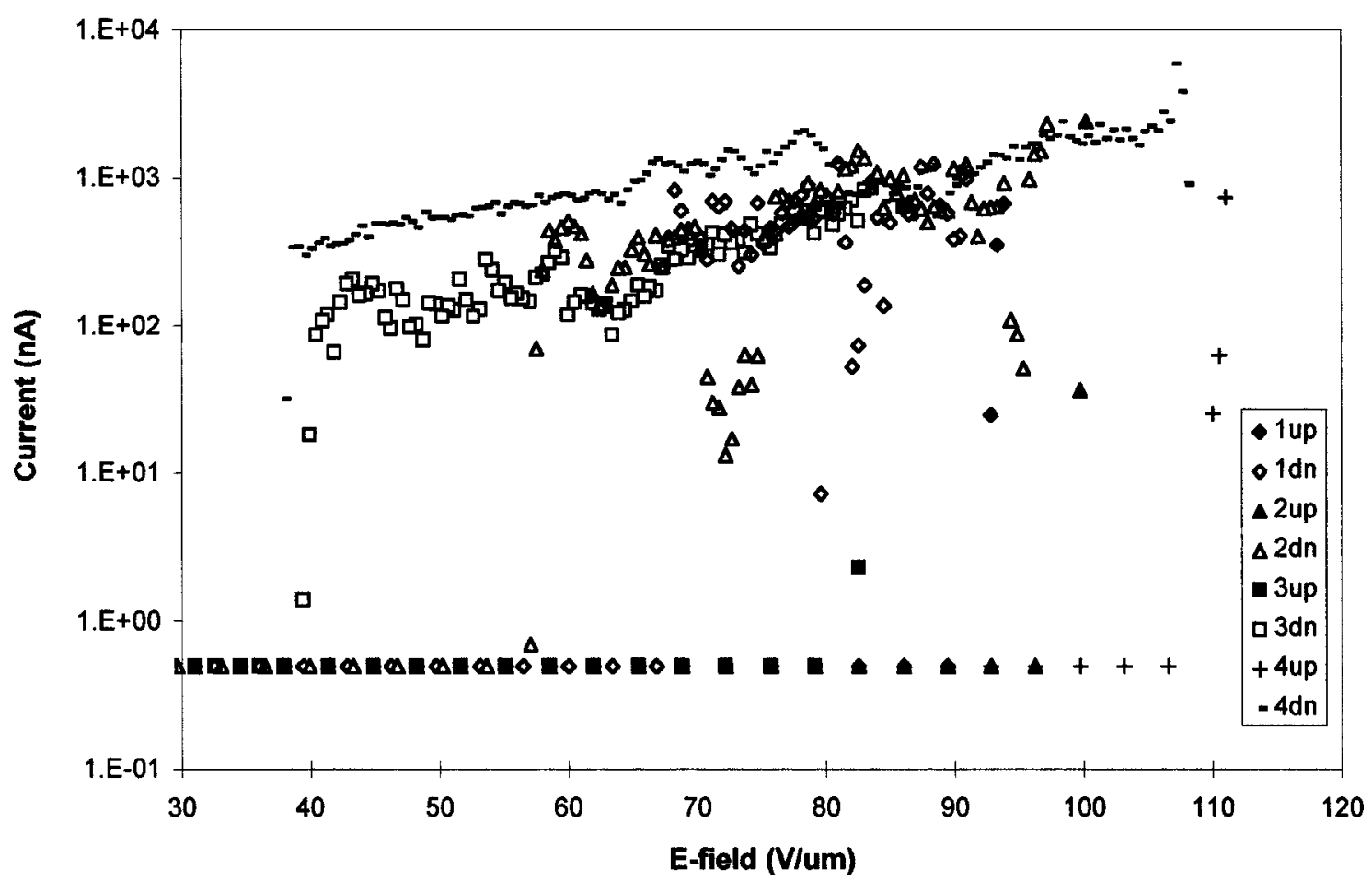

FIG. 3. Electron field emission vs macroscopic electric field for a $n^{+} a$-Si:H thin film. In this case there is an ohmic back contact to the $a$-Si:H film.

parameters. The barrier values obtained assuming a FowlerNordheim type conduction mechanism is one to two orders of magnitude too small which then manifests itself as a very low area of emission which is unrealistic for the cathodes analyzed in this article. It is well known that the electron affinity of $a$-Si:H is close to $4 \mathrm{eV}$, and therefore it is highly unlikely that the emission will be purely due to FowlerNordheim like tunneling at the front surface. The emission current measured appears to follow a space-charge-limited current more closely, which would be expected if the current-limiting process was purely due to a space charge in the film as proposed for DLC. ${ }^{4}$ In the case of the $a$-Si:H films instead of a heterojunction (as in DLC films in Ref. 4) at the back surface of the films, the reverse-biased Schottky barrier requires a high field across the film before electrons can be injected into the film by thermionic field emission. If these electrons gain enough energy in traveling across the thin films, they can surmount the vacuum barrier at the front surface of the cathode and $I-E$ currents as those observed would be measured. Not all the electrons will be able to leave the cathode and therefore with time a pool of electrons may collect at the front surface of the cathode, which will in time decrease the band bending and thus turn off the emission. We have experimentally observed the decrease and turning off of the emission current, with time. ${ }^{2,9}$ The time scales involved in this electron limiting process may be quite fast if the charge limiting was to occur as a result of modification of the fields and bands within the $a-\mathrm{Si}: \mathrm{H}$ as a result of the emission. On the other hand modification as a result of the occupation of electronic defect states by electrons would be much slower, and avoidable by pulsed mode operation of the device, with the surface charge extracted by means of a gated electrode. A possible band diagram when emission occurs in these films together with a more complete account of the charge limiting process will be published shortly.

The importance of the Schottky barrier at the back contact is highlighted in the emission characteristic shown in Fig. 3 for an $n^{+}$doped 100-nm-thick $a$-Si:H film. In this case the Schottky barrier is replaced by an ohmic contact which would not present a barrier at the back contact. The film is thus prevented from becoming fully depleted and the formation of a space charge layer that would lend itself to the creation of hot electrons is not allowed. It can be observed that the turn on threshold field of this cathode, even after conditioning, is not reproducible and is also significantly higher than the other films analyzed. Also, the current saturation level is lower than the other nondoped $a-\mathrm{Si}: \mathrm{H}$ thin films with a slope that is much less steep.

The electron emission process at low electric fields from flat cathodes is not properly understood as of yet. Emission models based purely on Fowler-Nordheim type tunneling from the front contact does not appear to fit the measured data unless very large beta factors or sharp surface features are assumed. ${ }^{10}$ It seems unlikely that such features could exist on a microscopic scale without it being observed in the scanning electron microscopic (SEM) study performed upon some of these $a$-Si:H cathodes. Films discussed in this article have shown mirror-quality surfaces before and after electron emission. On the other hand, by increasing the current threshold of the measurement system above a specified limit we can "stress" the films such that surface features of Fig. 4 result. In this case, $a$-Si:H cathodes were continuously run at very high current levels, for over $30 \mathrm{~h}$, such that they would become stressed, and then subject to a SEM study. The 


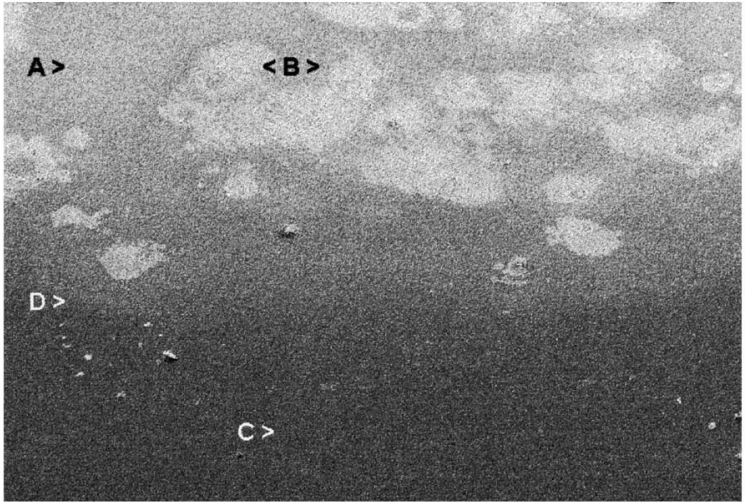

(a)

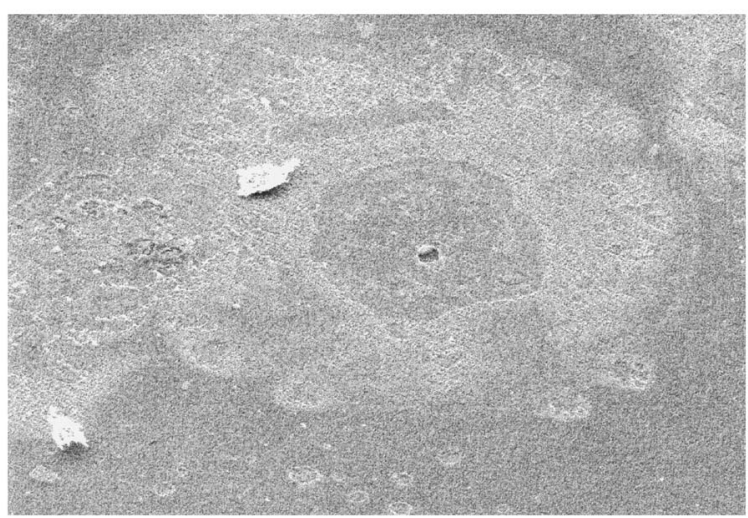

(b)

FIG. 4. SEM micrograph of a stressed $a$-Si:H film that has been operated for over 30 h continuously, above a critical current threshold. Region B in (a) has been expanded in (b). (a) Region of $850 \times 650 \mu \mathrm{m}$ and (b) an area of $150 \times 115 \mu \mathrm{m}$.

emission current levels of these diode structures were quite steady after the initial burn-in period, but as shown in Fig. 4 a "pock-marked" surface resulted. A comparison of the $I-E$ characteristics of the stressed and "unstressed" films has been published previously. ${ }^{2}$ A close up of feature B shown in Fig. 4(a), under a low accelerating voltage is shown in Fig. 4(b). An energy dispersive X-ray (EDX) study of the film revealed a surface that was still covered by a $\mathrm{Si}$ film, with $\mathrm{Cr}$ underneath. Under higher SEM accelerating voltages, the disruption to the underlying $\mathrm{Cr}$ layer is more visible. The boundary of the electron emission region shown by arrow $\mathrm{D}$ highlights the fact that there has been some change in the composition of the film for it to change in contrast. This could be due to hydrogen movement when emission takes place or charging of the cathode area after emission and cannot be elucidated in this study. The area shown by arrow A is typical for films that were not stressed, and the observed contrast between the emitted and nonemitted regions was not present. The features shown in Fig. 4(b) were less than $500 \mathrm{~nm}$ in height, and were composed primarily of $\mathrm{Si}$ and $\mathrm{Cr}$.

The variation of the threshold field with film thickness attributed to a space charge model may be explained by examining the voltages that are required to fully deplete a thin film. As the film thickness increases, the external voltage required to fully deplete the film increases in a nonlinear manner, which manifests itself as a higher electric field. On the other hand, if the film thickness is too low, the electrons that enter the conduction band of the $a-\mathrm{Si}: \mathrm{H}$ film do not gain enough energy in traversing down the electric field to overcome the surface potential barrier. These two conditions dictate that there will be an optimum film thickness window for electron emission. This phenomenon has been shown to occur for amorphous carbon thin films too. ${ }^{12}$

\section{CONCLUSION}

In this article we have shown that electron field emission can be observed from flat cathodes made of $a$-Si:H thin films, at relatively low electric fields. The Schottky barrier that is formed between the $\mathrm{Cr}$ back contact and the $a-\mathrm{Si}: \mathrm{H}$ is crucial for the operation of this device. The emission appears to be controlled primarily by the combined effect of the Schottky barrier and space-charge-induced band bending in the film. This is confirmed by the film thickness dependence of the field assisted electron emission characteristics of the diode structures. The $a$-Si thin films are candidates for cold cathodes as they can be incorporated easily into a field emission display in the form of active devices.

\section{ACKNOWLEDGMENTS}

R. D. F. would like to thank Philips Research Laboratories, Redhill for an industrial studentship. The authors also acknowledge the support received from EPSRC (GR/ L77638) for this work.

${ }^{1}$ R. A. Street, in Hydrogenated Amorphous Silicon (Cambridge University Press, Cambridge, 1991).

${ }^{2}$ S. R. P. Silva, R. D. Forrest, and J. M. Shannon, J. Non-Cryst. Solids 227-230, 1101 (1998).

${ }^{3}$ C. A. Spindt et al., IEEE Trans. Electron Devices 38, 2355 (1991).

${ }^{4}$ G. A. J. Amaratunga and S. R. P. Silva, Appl. Phys. Lett. 68, 2529 (1996).

${ }^{5}$ W. Zhu et al., Appl. Phys. Lett. 67, 1157 (1995).

${ }^{6}$ A. A. Talin et al., J. Vac. Sci. Technol. A 14, 2 (1996).

${ }^{7}$ J. Robertson, Mater. Res. Soc. Symp. Proc. 498, 197 (1998).

${ }^{8}$ J. Jaskie, E-MRS Meeting, Strasbourg, June 1998 (unpublished); MRS Bull. 21, 59 (1996).

${ }^{9}$ S. R. P. Silva, R. D. Forrest, D. A. Munindradasa, and G. A. J. Amaratunga, Diamond Relat. Mater. 7, 645 (1998).

${ }^{10}$ X. Shi, S. R. P. Silva, L. K. Cheah, and B. K. Tay, Appl. Phys. Lett. (in press).

${ }^{11}$ R. V. Latham, in High Voltage Vacuum Insulation (Academic, New York, 1981).

${ }^{12}$ R. D. Forrest, A. P. Burden, S. R. P. Silva, L. K. Cheah, and X. Shi, Appl. Phys. Lett. 73, 3784 (1998). 